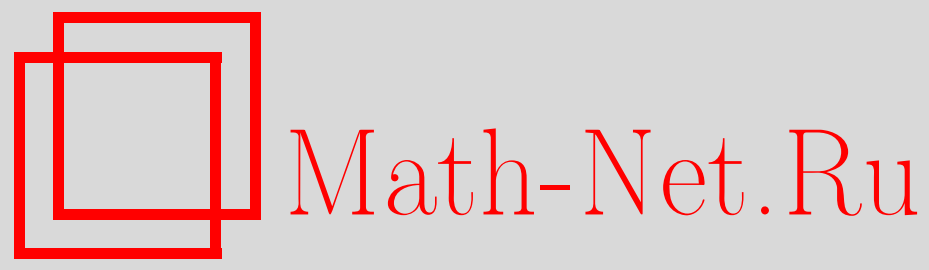

А. Г. Баскаков, Полугруппы разностных операторов в спектральном анализе линейных дифференциальных операторов, Функи. анализ и его прил., 1996, том 30, выпуск 3, 1-11

DOI: https://doi.org/10.4213/faa534

Использование Общероссийского математического портала MathNet.Ru подразумевает, что вы прочитали и согласны с пользовательским соглашением http://www.mathnet.ru/rus/agreement

Параметры загрузки:

IP : 54.209 .52 .79

26 апреля 2023 г., 15:42:41

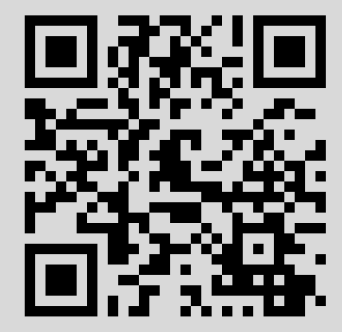


Функииональный анализ и его приложения

1996, т. 30, вып. 3, с. 1-11

УДК 517.9

\title{
Полугруппы разностных операторов \\ в спектральном анализе линейных дифференциальных операторов
}

\author{
(c) $1996 . \quad$ A. Г. БАСKAKOB ${ }^{1}$
}

Пусть $X$ - комплексное банахово пространство и End $X$ - банахова алгебра линейных ограниченных операторов, действующих в $X$. Символом $\mathscr{F}(\mathbb{R}, X)$ (или, короче, $\mathscr{F}$ ) обозначим одно из следующих банаховых пространств: $L_{p}=$ $L_{p}(\mathbb{R}, X) \quad(p \in[1, \infty])$ - пространство суммируемых со степенью $p$ (существенно ограниченных при $p=\infty)$ измеримых по Бохнеру на $\mathbb{R}=(-\infty, \infty)$ функций, принимающих значения в $X\left(\|\cdot\|_{p}\right.$ - норма в $\left.L_{p}(\mathbb{R}, X)\right), S_{p}=$ $S_{p}(\mathbb{R}, X)(p \in[1, \infty))$ - банахово пространство локально суммируемых со степенью $p$ измеримых на $\mathbb{R}$ функций со значениями в $X$, для которых конечна величина $\|x\|_{S_{p}}=\sup _{t \in \mathbb{R}}\left(\int_{0}^{1}\|x(s+t)\|^{p} d s\right)^{1 / p}\left(x \in S_{p}(\mathbb{R}, X)\right), C=C(\mathbb{R}, X)$ - подпространство непрерывных функций из $L_{\infty}(\mathbb{R}, X)$ и $C_{0}=C_{0}(\mathbb{R}, X)$ - подпространство функций из $C(\mathbb{R}, X)$ со свойством $\lim _{|t| \rightarrow \infty}\|x(t)\|=0$, $x \in C_{0}(\mathbb{R}, X)$.

Рассматривается семейство эволюционных операторов (пропогатор) $\mathscr{U}=$ $\{\mathscr{U}(t, s) ;-\infty<s \leqslant t<\infty\} \subset$ End $X$, т.е. предполагаются выполненными следующие условия:

1) семейство $\mathscr{U}$ сильно непрерывно на $\Delta=\left\{(t, s) \in \mathbb{R}^{2}: s \leqslant t\right\}$;

2) $\mathscr{U}(t, s) \mathscr{U}(s, \tau)=\mathscr{U}(t, \tau),-\infty<\tau \leqslant s \leqslant t<\infty$;

3) $\mathscr{U}(t, t)=I$ для любого $t \in \mathbb{R}$;

4) $\sup _{0 \leqslant t-s \leqslant 1}\|\mathscr{U}(t, s)\|=K<\infty$.

Семейству $\mathscr{U}$ сопоставляется линейный оператор

$$
\mathscr{L}_{\mathscr{U}}: D\left(\mathscr{L}_{\mathscr{U}}\right) \subset \mathscr{F}=\mathscr{F}(\mathbb{R}, X) \rightarrow \mathscr{F} .
$$

Его область определения $D\left(\mathscr{L}_{\mathscr{U}}\right)$ определяется так. Функция $x \in \mathscr{F}$ включается в $D\left(\mathscr{L}_{\mathscr{U}}\right)$, если существует функция $f \in \mathscr{F}$, такая, что для почти всех $s \leqslant t$ из $\mathbb{R}$ имеют место равенства

$$
x(t)=\mathscr{U}(t, s) x(s)-\int_{s}^{t} \mathscr{U}(t, \tau) f(\tau) d \tau .
$$

При этом мы полагаем $\mathscr{L}_{\mathscr{U}} x=f$.

\footnotetext{
${ }^{1}$ Работа выполнена при финансовой поддержке Международного научного фонда, гранты NZA000 и NZA300, и Российского фонда фундаментальных исследований, проект 95-01-00032.
} 
Таким образом, $\mathscr{L}_{\mathscr{U}}=-d / d t+A(t): D\left(\mathscr{L}_{\mathscr{U}}\right) \subset \mathscr{F} \rightarrow \mathscr{F}$ - абстрактный параболический оператор (см. [1, с. 165]), если $\mathscr{U}$ - семейство эволюционных операторов для линейного дифференщиального уравнения

$$
\dot{x}(t)=A(t) x(t), \quad t \in \mathbb{R},
$$

где $A(t): D(A(t)) \subset X \rightarrow X-$ семейство замкнутых линейных операторов, порождающих корректную задачу Коши (см. [2]).

Для исследования линейного оператора $\mathscr{L}_{\mathscr{U}}: D\left(\mathscr{L}_{\mathscr{U}}\right) \subset \mathscr{F} \rightarrow \mathscr{F}=\mathscr{F}(\mathbb{R}, X)$ в данной статье систематически используется полугруппа $\left\{T_{\mathscr{U}}(t), t \geqslant 0\right\}$ разностных операторов из банаховой алгебры End $\mathscr{F}$ вида

$$
\left(T_{\mathscr{U}}(t) x\right)(s)=\mathscr{U}(s, s-t) x(s-t), \quad x \in \mathscr{F}, s \in \mathbb{R}, t \geqslant 0 .
$$

Основные результаты статьи группируются вокруг следующих четырех теорем.

Теорема 1. Oператор $\mathscr{L}_{\mathscr{U}}$ является производящим оператором сильно непрерьвной полугруппь операторов $\left\{T_{\mathscr{U}}(t), t \geqslant 0\right\}$ в любом из банаховых пространств $L_{p}=L_{p}(\mathbb{R}, X), p \in[1, \infty), C_{0}=C_{0}(\mathbb{R}, X)$.

Теорема 2 (теорема об отображении спектра). Спектр $\sigma\left(\mathscr{L}_{\mathscr{U}}\right)$ оператора $\mathscr{L}_{\mathscr{U}}$ и спектр $\left\{\sigma\left(T_{\mathscr{U}}(t)\right)\right\}$ разностного оператора $T_{\mathscr{U}}, t>0$, свлзаны соотношением

$$
\sigma\left(T_{\mathscr{U}}(t)\right) \backslash\{0\}=\exp \sigma\left(\mathscr{L}_{\mathscr{U}}\right) t=\left\{\exp \lambda t: \lambda \in \sigma\left(\mathscr{L}_{\mathscr{U}}\right)\right\} .
$$

В частности, оператор $\mathscr{L}_{\mathscr{U}}$ обратим тогда и только тогда, когда обратим разностный оператор $\mathscr{D}_{\mathscr{U}}=I-T_{\mathscr{U}}(1)$, имеющий вид

$$
\left(\mathscr{D}_{\mathscr{U}} x\right)(s)=x(s)-\mathscr{U}(s, s-1) x(s-1), \quad x \in \mathscr{F}, s \in \mathbb{R} .
$$

Отметим, что утверждение теоремы 2 не имеет места для произвольной сильно непрерывной полугруппы операторов (см. [3, с. 676]).

Символом $\mathscr{F}(\mathbb{Z}, X)$ обозначим одно из следующих банаховых пространств двусторонних последовательностей векторов из банахова пространства $X$ :

$$
\begin{gathered}
l_{p}=l_{p}(\mathbb{Z}, X)=\left\{x: \mathbb{Z} \rightarrow X \mid\|x\|_{p}=\left(\sum_{n \in \mathbb{Z}}\|x(n)\|^{p}\right)^{1 / p}<\infty\right\}, \quad p \in[1, \infty), \\
l_{\infty}=l_{\infty}(\mathbb{Z}, X)=\left\{x: \mathbb{Z} \rightarrow X \mid\|x\|_{\infty}=\sup _{n \in \mathbb{Z}}\|x(n)\|<\infty\right\}, \\
c_{0}=C_{0}(\mathbb{Z}, X)=\left\{x \in l_{\infty} \mid \lim _{n \rightarrow \infty}\|x(n)\|=0\right\} .
\end{gathered}
$$

В следующей теореме используется пара банаховых пространств $(\mathscr{F}(\mathbb{R}, X)$, $\mathscr{F}(\mathbb{Z}, X))$, которая является одной из следующих: $\left(S_{p}, l_{\infty}\right),\left(L_{p}, l_{p}\right), p \in[1, \infty)$, $\left(C, l_{\infty}\right),\left(C_{0}, c_{0}\right)$.

Tеорема 3. Линейньй оператор $\mathscr{L}_{\mathscr{U}}: D\left(\mathscr{L}_{\mathscr{U}}\right) \subset \mathscr{F}(\mathbb{R}, X) \rightarrow \mathscr{F}(\mathbb{R}, X)$ обратим тогда и только тогда, когда обратим разностный оператор $\mathscr{D}_{0}: \mathscr{F}(\mathbb{Z}, X) \rightarrow \mathscr{F}(\mathbb{Z}, X)$, определяемьй равенствами

$$
\left(\mathscr{D}_{0} x\right)(n)=x(n)-\mathscr{U}(n, n-1) x(n-1), \quad x \in \mathscr{F}(\mathbb{Z}, X), n \in \mathbb{Z} .
$$


Будем говорить, что семейство эволюционных операторов $\{\mathscr{U}(t, s), s \leqslant t\}$ из алгебры End $X$ допускает экспоненциальную дихотомию на $\mathbb{R}$ с показателем $\beta>0$ и коэффищиентом $M>0$, если существует ограниченная сильно непрерывная проекторнозначная функция $P: \mathbb{R} \rightarrow \operatorname{End} X$, такая, что: 1$) \mathscr{U}(t, s) P(s)$ $=P(t) \mathscr{U}(t, s)$ при $t \geqslant s$ из $\mathbb{R} ; 2)\|\mathscr{U}(t, s) P(s)\| \leqslant M \exp (-\beta(t-s))$ при $t \geqslant s$ из $\mathbb{R} ; 3)$ при $t \geqslant s$ сужение $\mathscr{U}(t, s) \mid \operatorname{Im} Q(s)$ оператора $\mathscr{U}(t, s)$ на образ $\operatorname{Im} Q(s)$ проектора $Q(s)=I-P(s)$ (здесь и далее символом $I$ обозначается тождественный оператор) является изоморфизмом подпространств $\operatorname{Im} Q(s)$ и $\operatorname{Im} Q(t)$ (определим оператор $\mathscr{U}(s, t)$ как обратное отображение из $\operatorname{Im} Q(t)$ в $\operatorname{Im} Q(s)) ; 4)\|\mathscr{U}(t, s) Q(s)\| \leqslant M \exp \beta(t-s)$ при $s \geqslant t$ (нормы берутся в End $X$ и оператор $\mathscr{U}(t, s) Q(s)$ рассматривается как элемент пространства End $X)$.

Если $P=0$ или $Q=0$, то говорят, что для $\mathscr{U}$ имеет место тривиальная дихотомия.

ТЕОРемА 4. Имеют место следуюшие утверждения:

1) линейный оператор $\mathscr{L}_{\mathscr{U}}: D\left(\mathscr{L}_{\mathscr{U}}\right) \subset \mathscr{F} \rightarrow \mathscr{F}$ обратим тогда и только тогда, когда семейство $\mathscr{U}$ допускает экспоненииальную дихотомию;

2) спектр $\sigma\left(\mathscr{L}_{\mathscr{U}}\right)$ оператора $\mathscr{L}_{\mathscr{U}}$ не зависит от выбора пространства $\mathscr{F}(\mathbb{R}, X)$.

Сформулированные результаты были анонсированы в работе [4]. Исследование дифференциальных уравнений с использованием разностных операторов вида (3)-(4) осуществлялось в работах [1, 5-9].

\section{§1. Доказательство основных утверждений}

При доказательстве многих результатов используется следующее (вытекающее из равенств (1))

ЗАМЕЧАНИЕ 1. Для любого комплексного числа $\lambda \in \mathbb{C}$ оператор $\mathscr{L}_{\mathscr{U}}+\lambda I$ представим в виде $\mathscr{L}_{\mathscr{U}}+\lambda I=\mathscr{L}_{\mathscr{U}(\lambda)}$, где семейство эволюционных операторов $\mathscr{U}(\lambda)$ из алгебры End $X$ имеет вид $\mathscr{U}(\lambda)(t, s)=\exp \lambda(t-s) \mathscr{U}(t, s), s \leqslant t$.

Непосредственно из определения экспоненциальной дихотомии семейства $\mathscr{U}$ следует, что имеет место (см. [1, с. 167]) следующая

ЛЕмма 1. Пусть семейство И обладает экспоненииальной дихотомией на $\mathbb{R}$. Тогда линейньй оператор $\mathscr{L}_{\mathscr{U}}: D\left(\mathscr{L}_{\mathscr{U}}\right) \subset \mathscr{F}=\mathscr{F}(\mathbb{R}, X) \rightarrow \mathscr{F}$ (непрерылвно) обратим и обратныи $\mathscr{L}_{\mathscr{U}}^{-1} \in \operatorname{End} \mathscr{F}$ определяется формулой

$$
\left(\mathscr{L}_{\mathscr{U}}^{-1} f\right)(t)=\int_{\mathbb{R}} G(t, s) f(s) d s, \quad f \in \mathscr{F}, t \in \mathbb{R},
$$

где функиия Грина $G: \mathbb{R}^{2} \rightarrow$ End $X$ имеет вид

$$
G(t, s)= \begin{cases}-\mathscr{U}(t, s) P(s), & s \leqslant t \\ \mathscr{U}(t, s) Q(s), & s>t\end{cases}
$$

Отметим, что для $\mathscr{F}=C, C_{0}$ в монографии $[1$, гл. Х $]$ установлена эквивалентность обратимости оператора $\mathscr{L}_{\mathscr{U}}$ условию экспоненциальной дихотомии семейства $\mathscr{U}$.

СлЕдСТВиЕ. Существует такое число $\alpha \in \mathbb{R}$, что оператор $\mathscr{L}_{\mathscr{U}}-\alpha I$ : $D\left(\mathscr{L}_{\mathscr{U}}\right) \subset \mathscr{F} \rightarrow \mathscr{F}$ обратим. 
Для доказательства достаточно заметить, что из свойств 3) и 4) семейства $\mathscr{U}$ следует существование постоянных $C>0$ и $\beta_{0} \in \mathbb{R}$, таких, что $\|\mathscr{U}(t, s)\| \leqslant$ $C \exp \beta_{0}(t-s)$ при $s \leqslant t$ из $\mathbb{R}$. Если $\alpha>\beta_{0}$, то в силу замечания 1 имеет место равенство $\mathscr{L}_{\mathscr{U}}-\alpha I=\mathscr{L}_{\mathscr{U}(-\alpha)}$. Для семейства $\mathscr{U}(-\alpha)$ имеет место тривиальная дихотомия $\left(\|\mathscr{U}(-\alpha)(t, s)\| \leqslant C \exp \left(\beta_{0}-\alpha\right)(t-s), s \leqslant t\right)$, и поэтому оператор $\mathscr{L}_{\mathscr{U}}-\alpha I$ обратим.

ДОКАЗАТЕЛЬСТВо ТЕОремЫ 1 . Пусть $\mathscr{A}$ - производящий оператор сильно непрерывной полугруппы операторов $\left\{T_{\mathscr{U}}(t), t \geqslant 0\right\}$, определяемой формулой $(2)$. Достаточно доказать, что существует число $\alpha \in \mathbb{R}$, принадлежащее пересечению $\rho(\mathscr{A}) \cap \rho\left(\mathscr{L}_{\mathscr{U}}\right)$ резольвентных множеств $\rho(\mathscr{A})$ и $\rho\left(\mathscr{L}_{\mathscr{U}}\right)$ операторов $\mathscr{A}$ и $\mathscr{L}_{\mathscr{U}}$ соответственно и такое, что операторы $(\mathscr{A}-\alpha I)^{-1},\left(\mathscr{L}_{\mathscr{U}}-\alpha I\right)^{-1}$ совпадают. Существование такого числа $\alpha$ вытекает из следствия леммы 1 и того факта, что $\mathscr{A}$ - производящий оператор сильно непрерывной полугруппы операторов (см. [3]). Рассматривая, если необходимо, вместо операторов $\mathscr{A}$ и $\mathscr{L}_{\mathscr{U}}$ операторы $\mathscr{A}-\alpha I$ и $\mathscr{L}_{\mathscr{U}}-\alpha I$, без ограничения общности можно далее считать, что $\alpha=0$, т. е. в этом случае $\left\|T_{\mathscr{U}}(t)\right\|=\sup _{s \in \mathbb{R}}\|\mathscr{U}(s, s-t)\| \leqslant$ Const $\exp (-\gamma t), t \geqslant 0$, для некоторого $\gamma>0$. Следовательно, для семейства $\mathscr{U}$ имеет место тривиальная дихотомия, и потому из леммы 1 получаем представление

$$
\left(\mathscr{L}_{\mathscr{U}}^{-1} f\right)(s)=-\int_{-\infty}^{s} \mathscr{U}(s, \tau) f(\tau) d \tau, \quad f \in \mathscr{F} .
$$

С другой стороны, для оператора $\mathscr{A}^{-1}$ имеют место следующие равенства (см. $[3$, c. 354]):

$$
\begin{aligned}
\left(\mathscr{A}^{-1} f\right)(s) & =-\int_{0}^{\infty}\left(T_{\mathscr{U}}(t) f\right)(s) d t=-\int_{0}^{\infty} \mathscr{U}(s, s-t) f(s-t) d t \\
& =-\int_{-\infty}^{s} \mathscr{U}(s, \tau) f(\tau) d \tau=\left(\mathscr{L}_{\mathscr{U}}^{-1} f\right)(s), \quad f \in \mathscr{F} .
\end{aligned}
$$

Теорема доказана.

В следующих двух леммах используется принятое в теореме 3 соглашение относительно пары $(\mathscr{F}(\mathbb{R}, X), \mathscr{F}(\mathbb{Z}, X))$ банаховых пространств.

Лемма 2. Из обратимости оператора $\mathscr{L}_{\mathscr{U}}: D\left(\mathscr{L}_{\mathscr{U}}\right) \subset \mathscr{F}(\mathbb{R}, X) \rightarrow \mathscr{F}(\mathbb{R}, X)$ следует обратимость разностного оператора $\mathscr{D}_{0}$ (см. формулу (4)), причем имеет место оченка

$$
\left\|\mathscr{D}_{0}^{-1}\right\| \leqslant 1+3 K+\frac{9}{2} K^{2}\left\|\mathscr{L}_{\mathscr{U}}^{-1}\right\|, \quad K=\sup _{0 \leqslant t-s \leqslant 1}\|\mathscr{U}(t, s)\| .
$$

ДокАЗАтЕльство. Пусть оператор $\mathscr{L}_{\mathscr{\ell}}$ обратим. Докажем, что оператор $\mathscr{D}_{0}$ обратим и обратный имеет вид

$$
\mathscr{D}_{0}^{-1} x=\tilde{y}+x, \quad x \in \mathscr{F}(\mathbb{Z}, X),
$$

где через $\tilde{y}$ обозначено сужение функции $y=\mathscr{L}_{\mathscr{U}}^{-1} B x \in C(\mathbb{R}, X)$ на $\mathbb{Z}$ (включение $D\left(\mathscr{L}_{\mathscr{U}}\right) \subset C(\mathbb{R}, X)$ следует из равенств $\left.(1)\right)$ и линейный оператор $B: \mathscr{F}(\mathbb{Z}, X) \rightarrow \mathscr{F}(\mathbb{R}, X)$ определен равенствами

$$
(B x)(s)=-\varphi(s) \mathscr{U}(s, n-1) x(n-1), \quad x \in \mathscr{F}(\mathbb{Z}, X), n \in \mathbb{Z}, s \in[n-1, n] .
$$


Здесь $\varphi: \mathbb{R} \rightarrow \mathbb{R}$ - периодическая периода 1 функция, причем $\varphi(s)=6 s(1-s)$ для $s \in[0,1]$. Оператор $B$ корректно определен, ограничен, и $\|B\| \leqslant \frac{3}{2} K$.

Вначале докажем инъективность оператора $\mathscr{D}_{0}$. Если $x_{0} \in \operatorname{Ker} \mathscr{D}_{0}=$ $\left\{x \in \mathscr{F}(\mathbb{Z}, X): \mathscr{D}_{0} x=0\right\}$, то $x_{0}(n)=\mathscr{U}(n, n-1) x_{0}(n-1)$ для любого $n \in \mathbb{Z}$ и функция $x \in \mathscr{F}(\mathbb{R}, X)$, определенная равенствами

$$
x(t)=\mathscr{U}(t, n) x_{0}(n), \quad t \in[n, n+1], n \in \mathbb{Z},
$$

принадлежит ядру $\operatorname{Ker} \mathscr{L}_{\mathscr{U}}$ оператора $\mathscr{L}_{\mathscr{U}}$. Поэтому $x=0$ и, следовательно, $x_{0}=0$.

Докажем сюръективность оператора $\mathscr{D}_{0}$. Пусть $g \in \mathscr{F}(\mathbb{Z}, X), f=B g \in$ $\mathscr{F}(\mathbb{R}, X)$ и $x=\mathscr{L}^{-1} f \in D(\mathscr{L}) \subset C(\mathbb{R}, X)$. Тогда из равенств (1) получаем

$$
\begin{aligned}
x(n) & =\mathscr{U}(n, n-1) x(n-1)+\int_{n-1}^{n} \varphi(s) \mathscr{U}(n, s) \mathscr{U}(s, n-1) g(n-1) d s \\
& =\mathscr{U}(n, n-1) x(n-1)+\mathscr{U}(n, n-1) g(n-1), \quad n \in \mathbb{Z} .
\end{aligned}
$$

Следовательно, $\mathscr{D}_{0}(\tilde{x}+g)=g$, если только будет установлено, что $\tilde{x} \in \mathscr{F}(\mathbb{Z}, X)$. Из равенств (1) следуют оценки

$$
\|\tilde{x}(n)\|=\|x(n)\| \leqslant \frac{3}{2} K(\|x(s)\|+\|g(n-1)\|), \quad s \in[n-1, n], n \in \mathbb{Z} .
$$

Поэтому $\tilde{x} \in l_{\infty}(\mathbb{Z}, X)$, если $\mathscr{F}=L_{\infty}$ или $\mathscr{F}=C$, причем $\tilde{x} \in C_{0}(\mathbb{Z}, X)$ при $\mathscr{F}=C_{0}(\mathbb{R}, X)$. Из $(7)$ получаем

$$
\|\tilde{x}\|_{\infty}=\sup _{n \in \mathbb{Z}}\|x(n)\| \leqslant \frac{3}{2} K\left(\|x\|_{\infty}+\|g\|_{\infty}\right) .
$$

Пусть теперь $\mathscr{F}(\mathbb{R}, X)=L_{p}(\mathbb{R}, X), p \in[1, \infty)$, или $\mathscr{F}(\mathbb{R}, X)=S_{p}(\mathbb{R}, X)$, $p \in[1, \infty)$. Поскольку $(a+b)^{p} \leqslant 2^{p-1}\left(a^{p}+b^{p}\right)$ для любых $a, b \geqslant 0$, то, интегрируя по отрезку $[n, n+1]$ обе части неравенства $(7)$, возведенные в степень $p$, получаем неравенства

$$
\|x(n)\|^{p} \leqslant \frac{1}{2}(3 K)^{p}\left(\int_{n-1}^{n}\|x(s)\|^{p} d s+\|g(n-1)\|^{p}\right), \quad n \in \mathbb{Z} .
$$

Поэтому при $\mathscr{F}(\mathbb{R}, X)=L_{p}(\mathbb{R}, X)$ имеет место оценка

$$
\|\tilde{x}\|_{p} \leqslant 3 K\left(\|x\|_{p}+\|g\|_{p}\right) .
$$

Если $\mathscr{F}(\mathbb{R}, X)=S_{p}(\mathbb{R}, X)$, то из $(9)$ следует, что

$$
\|\tilde{x}\|_{\infty} \leqslant 3 K\left(\|x\|_{S_{p}}+\|g\|_{\infty}\right) .
$$

Таким образом, из оценок (7)-(11) получаем, что при любом выборе пространства $\mathscr{F}(\mathbb{R}, X)$ функция $\tilde{x}$ принадлежит пространству $\mathscr{F}(\mathbb{Z}, X)$. Следовательно, оператор $\mathscr{D}_{0}$ обратим, $\tilde{x}+g=\mathscr{D}_{0}^{-1} g$ и из приведенных оценок вытекает, что

$$
\begin{aligned}
\left\|\mathscr{D}_{0}^{-1} g\right\| & \leqslant\|\tilde{x}\|+\|g\| \leqslant 3 K\left(\|x\|_{\mathscr{F}}+\|g\|\right)+\|g\| \\
& \leqslant 3 K\left(\left\|\mathscr{L}^{-1}\right\|\|B\|\|g\|+\|g\|\right)+\|g\|=\left(1+3 K+\frac{9}{2} K^{2}\left\|\mathscr{L}^{-1}\right\|\right)\|g\| .
\end{aligned}
$$


Лемма 3. Из обратимости разностного оператора $\mathscr{D}_{0} \in$ End $\mathscr{F}(\mathbb{Z}, X)$ следует обратимость оператора $\mathscr{L}_{\mathscr{U}}: D\left(\mathscr{L}_{\mathscr{U}}\right) \subset \mathscr{F} \rightarrow \mathscr{F}=\mathscr{F}(\mathbb{R}, X)$.

ДокаЗАТЕЛЬСтво. Пусть оператор $\mathscr{D}_{0}$ (см. формулу (4)) обратим. Из его инъективности следует инъективность оператора $\mathscr{L}_{\mathscr{U}}$ (если $\mathscr{L}_{\mathscr{U}} x=0$, то сужение $\tilde{x}$ функции $x$ на $\mathbb{Z}$ принадлежит $\operatorname{Ker} \mathscr{D}_{0}$; поэтому из равенств $x(t)=$ $\mathscr{U}(t, n) x(n)=0$ для любого $t \in[n, n+1], n \in \mathbb{Z}$, следует, что $x=0)$.

Докажем сюръективность оператора $\mathscr{L}_{\mathscr{U}}$. Для произвольной функции $f \in \mathscr{F}$ рассмотрим функцию $f_{d} \in \mathscr{F}(\mathbb{Z}, X)$ вида $f_{d}(n)=-\int_{n}^{n+1} \mathscr{U}(n, \tau) f(\tau) d \tau, n \in \mathbb{Z}$. Тогда существует функция $x_{0} \in \mathscr{F}(\mathbb{Z}, X)$, такая, что $\mathscr{D} x_{0}=f_{d}$. Непосредственной проверкой легко убедиться в том, что функция $x \in \mathscr{F}$, определенная на каждом промежутке $[n, n+1](n \in \mathbb{Z})$ соотношениями

$$
x(s)=\mathscr{U}(s, n) x_{0}(n)-\int_{n}^{s} \mathscr{U}(s, \tau) f(\tau) d \tau, \quad s \in[n, n+1],
$$

принадлежит $D\left(\mathscr{L}_{\mathscr{U}}\right)$ и $\mathscr{L}_{\mathscr{U}} x=f$ (т. е. выполнены равенства $\left.(1)\right)$. Лемма доказана.

ДОКАЗАТЕЛЬСТВО ТЕОРЕМЫ 3 непосредственно следует из лемм 2 и 3.

ДОКАЗАТЕЛЬСТВО ТЕОРЕМЫ 4. Достаточность условия экспоненциальной дихотомии семейства $\mathscr{U}$ установлена в лемме 1.

Пусть теперь оператор $\mathscr{L}_{\mathscr{U}}$ обратим. Из теоремы 3 следует обратимость соответствующего разностного оператора $\mathscr{D}_{0} \in \operatorname{End} \mathscr{F}(\mathbb{Z}, X)$. Из [11, теорема 3$]$ получаем, что оператор $\mathscr{D}_{0}$ обратим в банаховом пространстве $l_{\infty}(\mathbb{Z}, X)$. Еще раз используя теорему 3 , приходим к выводу о том, что оператор $\mathscr{L}_{\mathscr{U}}$ обратим в пространстве $C(\mathbb{R}, X)$ и, следовательно, из работы $[1$, гл. $\mathrm{X}]$ получаем, что семейство $\mathscr{U}$ допускает экспоненциальную дихотомию. Теорема доказана.

ЗАмечАнИЕ 2. Доказательство необходимости приводимого в теореме 4 условия можно изложить иначе, рассматривая семейство обратимых операторов вида $\left\{S(\alpha) \mathscr{L}_{\mathscr{U}} S(-\alpha) ; \alpha \in \mathbb{R}\right\}$, где $\{S(\alpha) ; \alpha \in \mathbb{R}\}$ - группа изометрических операторов сдвигов функщий из $\mathscr{F}(\mathbb{R}, X)((S(\alpha) x)(t)=x(t+\alpha), x \in \mathscr{F})$. Из леммы 2 будет следовать равномерная обратимость соответствующего семейства разностных операторов

$$
\left(\mathscr{D}_{\alpha} x\right)(n)=x(n)-\mathscr{U}(n+\alpha, n+\alpha-1) x(n-1), \quad \alpha \in \mathbb{R}, x \in \mathscr{F}(\mathbb{Z}, X) .
$$

Значит, они допускают дискретную экспоненциальную дихотомию на $\mathbb{Z}$ (см. [6, c. 250]). Экспоненциальная дихотомия семейства $\mathscr{U}$ будет следовать из [6, с. 251].

ДоКАЗАТЕЛЬСТво ТЕОРЕмЫ 2. Из теоремы 3 статьи [11] следует, что доказательство теоремы достаточно провести для случая, когда $\mathscr{F}(\mathbb{R}, X)$ совпадает с одним из банаховых пространств $L_{p}(\mathbb{R}, X), p \in[1, \infty), C_{0}(\mathbb{R}, X)$, на которых полугруппа $\left\{T_{\mathscr{U}}(t), t \geqslant 0\right\}$ сильно непрерывна.

Включение $\exp \sigma\left(\mathscr{L}_{\mathscr{U}}\right) t \subset \sigma\left(T_{\mathscr{U}}(t)\right) \backslash\{0\}$ известно (оно верно для произвольной сильно непрерывной полугруппы линейных операторов [3, гл. XVI]). Включение в другую сторону следует из формулы

$$
\left(\left(T_{\mathscr{U}}(t)-I\right)^{-1} x\right)(s)=\sum_{k \in \mathbb{Z}} G(s, s+k t) x(s+k t), \quad t>0,
$$


которая имеет место при условии обратимости оператора $\mathscr{L}_{\mathscr{U}}(G-$ функция Грина, определенная формулой (6)). Действительно, при любых $x \in \mathscr{F}(\mathbb{R}, X)$, $s \in \mathbb{R}$ и $t>0$ верны следующие равенства:

$$
\begin{aligned}
& \left(T_{\mathscr{U}}(t)-I\right)\left(\sum_{k \in \mathbb{Z}} G(s, s+k t) x(s+k t)\right) \\
& =\mathscr{U}(s, s-t)\left[-\sum_{k \leqslant 0} \mathscr{U}(s-t, s+(k-1) t) P(s+(k-1) t) x(s+(k-1) t)\right. \\
& \left.\quad+\sum_{k \geqslant 1} \mathscr{U}(s-t, s+(k-1) t) Q(s+(k-1) t) x(s+(k-1) t)\right] \\
& \quad+\sum_{k \leqslant 0} \mathscr{U}(s, s+k t) P(s+k t) x(s+k t)-\sum_{k \geqslant 1} \mathscr{U}(s, s+k t) Q(s+k t) x(s+k t) \\
& =\mathscr{U}(s, s)(P(s)+Q(s)) x(s)=x(s) .
\end{aligned}
$$

Аналогично проверяется, что оператор, определенный формулой (12), является также и левым обратным для оператора $T_{\mathscr{U}}(t)-I$. Итак, доказано, что если $0 \notin \sigma\left(\mathscr{L}_{\mathscr{U}}\right)$, то $1 \notin \sigma\left(T_{\mathscr{U}}(t)\right)$ для любого $t>0$. Случай $\lambda \notin \sigma\left(\mathscr{L}_{\mathscr{U}}\right)$ сводится к рассмотренному с использованием семейства $\mathscr{U}(\lambda)$ (см. замечание 1) и оператора $\mathscr{L}_{\mathscr{U}(\lambda)}=\mathscr{L}_{\mathscr{U}}-\lambda I$ и с учетом того факта, что $\mathscr{L}_{\mathscr{U}(\lambda)}$ является производящим оператором полугруппы операторов $\left\{T_{\mathscr{U}}(t) \exp (-\lambda t), t \geqslant 0\right\}$. Второе утверждение теоремы следует из первого утверждения и леммы 1. Теорема доказана.

СЛЕдСТВИЕ. Если оператор $\mathscr{L}_{\mathscr{U}}$ обратим, mo оператор $\mathscr{D}_{0}^{-1} \in \operatorname{End} \mathscr{F}(\mathbb{Z}, X)$ имеет вид

$$
\left(\mathscr{D}_{0}^{-1} x\right)(n)=\sum_{m \in \mathbb{Z}} G(n, m) x(m), \quad x \in \mathscr{F}(\mathbb{Z}, X), n \in \mathbb{Z} .
$$

Teорема 5. Cnектр $\sigma\left(\mathscr{L}_{\mathscr{U}}\right)$ оператора $\mathscr{L}_{\mathscr{U}}: D\left(\mathscr{L}_{\mathscr{U}}\right) \subset \mathscr{F} \rightarrow \mathscr{F}$ являетсл объединением некоторого семейства прямых из $\mathbb{C}$, параллельньх мнимой оси $i \mathbb{R}$, и содержит прямую

$$
\left\{\lambda \in \mathbb{C}: \operatorname{Re} \lambda=\chi_{+}(\mathscr{U})\right\}, \quad \chi_{+}(\mathscr{U})=\lim _{\tau \rightarrow \infty} \frac{1}{\tau} \ln \sup _{s \in \mathbb{R}}\|\mathscr{U}(s, s-\tau)\|,
$$

причем $\sup _{\lambda \in \sigma\left(\mathscr{L}_{\mathscr{U}}\right)} \operatorname{Re} \lambda=\chi_{+}(\mathscr{U})$. Если размерность $\operatorname{dim} X$ банахова пространства $X$ конечна, то имеет место следуюшее представление для $\sigma\left(\mathscr{L}_{\mathscr{U}}\right)$ :

$$
\sigma\left(\mathscr{L}_{\mathscr{U}}\right)=\bigcup_{k=1}^{m}\left\{\lambda \in \mathbb{C}: \alpha_{k} \leqslant \operatorname{Re} \lambda \leqslant \beta_{k}\right\}
$$

гдe $-\infty \leqslant \alpha_{1} \leqslant \beta_{1}<\alpha_{2} \leqslant \beta_{2}<\cdots<\alpha_{m} \leqslant \beta_{m}=\chi_{+}(\mathscr{U})<\infty, m \leqslant \operatorname{dim} X$.

В частности, спектр скалярного дифференциального оператора $\mathscr{L}=-d / d t+$ $a(t): D(\mathscr{L}) \subset \mathscr{F}(\mathbb{R}, \mathbb{C}) \rightarrow \mathscr{F}(\mathbb{R}, \mathbb{C})$, где $a \in S_{1}(\mathbb{R}, \mathbb{C})$, совпадает с множеством

$$
\left\{\lambda \in \mathbb{C}: \lim _{\tau \rightarrow \infty} \frac{1}{\tau} \inf _{s \in \mathbb{R}} \int_{s}^{s+\tau} \operatorname{Re} a(\alpha) d \alpha \leqslant \operatorname{Re} \lambda \leqslant \lim _{\tau \rightarrow \infty} \frac{1}{\tau} \sup _{s \in \mathbb{R}} \int_{s}^{s+\tau} \operatorname{Re} a(\alpha) d \alpha\right\} .
$$


ДоКАЗАТЕЛЬСТВО. В силу теоремы 2 достаточно рассмотреть разностный оператор $T_{\mathscr{U}}(1) \in$ End $\mathscr{F}(\mathbb{R}, X)$ и получить соответствующие утверждения о его спектре (как объединении концентрических окружностей). Рассмотрим группу изометрических линейных операторов $\{V(\lambda), \lambda \in \mathbb{R}\}$ из банаховой алгебры End $\mathscr{F}(\mathbb{R}, X)$, имеющих вид

$$
(V(\lambda) x)(t)=(\exp i \lambda t) x(t), \quad x \in \mathscr{F}(\mathbb{R}, X), t, \lambda \in \mathbb{R} .
$$

Из равенств $V(\lambda) T_{\mathscr{U}}(1) V(-\lambda)=(\exp i \lambda) T_{\mathscr{U}}(1), \lambda \in \mathbb{R}$, означающих подобие оператора $T_{\mathscr{U}}(1)$ операторам $\gamma T_{\mathscr{U}}(1), \gamma \in \mathbb{T}$, следует, что спектр $\sigma\left(T_{\mathscr{U}}(1)\right)$ оператора $T_{\mathscr{U}}(1)$ является объединением семейства окружностей с центром в точке 0 .

Допустим, что некоторая окружность $S(\alpha)=\{z \in \mathbb{C}:|z|=\alpha\}, \alpha>0$, принадлежит резольвентному множеству $\rho\left(T_{\mathscr{U}}(1)\right)$ оператора $T_{\mathscr{U}}(1)$, и пусть $\sigma\left(T_{\mathscr{U}}(1)\right)$ является объединением двух спектральных множеств

$$
\sigma_{+}=\left\{\lambda \in \sigma\left(T_{\mathscr{U}}(1)\right):|\lambda|>\alpha\right\}, \quad \sigma_{-}=\left\{\lambda \in \sigma\left(T_{\mathscr{U}}(1)\right):|\lambda|<\alpha\right\} .
$$

Пусть $P\left(\sigma_{+}\right)$и $P\left(\sigma_{-}\right)$- соответствуюшие проекторы Рисса (так что $I=$ $\left.P\left(\sigma_{+}\right)+P\left(\sigma_{-}\right)\right)$.

Без ограничения общности можно считать, что $\alpha=1$. В этом случае $\mathscr{L}_{\mathscr{U}}-$ обратимый оператор и, согласно теореме 4 , для семейства $\mathscr{U}$ имеет место экспоненциальная дихотомия. Представим проектор $P\left(\sigma_{-}\right)$в виде (см. $[10$, с. 33$\left.]\right)$

$$
P\left(\sigma_{-}\right)=\frac{1}{2 \pi i} \int_{\mathbb{T}}\left(\gamma I-T_{\mathscr{U}}(1)\right)^{-1} d \gamma=\frac{1}{2 \pi} \int_{0}^{2 \pi}\left(e^{i \varphi} I-T_{\mathscr{U}}(1)\right)^{-1} e^{i \varphi} d \varphi .
$$

Используя формулу (аналог формулы (13))

$$
\left(\left(e^{i \varphi} I-T_{\mathscr{U}}(1)\right)^{-1} x\right)(s)=\sum_{k \in \mathbb{Z}} e^{-i(k+1) \varphi} G(s, s+k) x(s+k),
$$

после интегрирования выражения для $P\left(\sigma_{-}\right)$получаем, что проектор $P\left(\sigma_{-}\right)$ является оператором умножения вида

$$
\left(P\left(\sigma_{-}\right) x\right)(s)=G(s, s) x(s)=P(s) x(s), \quad x \in \mathscr{F}, s \in \mathbb{R},
$$

где $P: \mathbb{R} \rightarrow$ End $X$ - сильно непрерывная проекторнозначная функция, участвующая в определении экспоненциальной дихотомии семейства $\mathscr{U}$.

Пусть $\operatorname{dim} X<\infty$; тогда функция $P$ непрерывна (в равномерной операторной топологии), и потому размерность всех образов проекторов $P(s), s \in \mathbb{R}$, не зависит от $s \in \mathbb{R}$. Отсюда и из вида проекторов Рисса следует, что число связных спектральных компонент у множества $\sigma\left(T_{\mathscr{U}}(1)\right)$ не превосходит числа $\operatorname{dim} X$. Поэтому из теоремы 2 следует, что имеет место представление вида (14).

Утверждение теоремы о включении прямой $\chi(\mathscr{U})+i \mathbb{R}$ в множество $\sigma\left(T_{\mathscr{U}}(1)\right)$ и равенство $\chi_{+}(\mathscr{U})=\sup _{\lambda \in \sigma\left(\mathscr{L}_{\mathscr{U}}\right)} \operatorname{Re} \lambda$ непосредственно следуют из теоремы 2 и равенства

$$
r\left(T_{\mathscr{U}}(1)\right)=\lim _{n \rightarrow \infty}\left\|T_{\mathscr{U}}(n)\right\|^{1 / n}=\lim _{n \rightarrow \infty} \sup _{s \in \mathbb{R}}\|\mathscr{U}(s, s-n)\|^{1 / n}=\exp \chi_{+}(\mathscr{U}),
$$

вытекающего из формулы Гельфанда для спектрального радиуса [3, с. 138]. 
Если $\mathscr{L}=-d / d t+a(t)$, то $\operatorname{dim} X=1$ и поэтому множество $\sigma\left(T_{\mathscr{U}}(1)\right)$ связно. В этом случае $\mathscr{U}(s, s-\tau)=\exp \int_{s-\tau}^{s} a(\lambda) d \lambda, s \in \mathbb{R}, \tau \geqslant 0$. Поскольку оператор $T_{\mathscr{U}}(1)$ обратим и обратный к нему совпадает с оператором $T_{\mathscr{U}}(-1)$, то, применяя к $T_{\mathscr{U}}(-1)$ формулу Гельфанда, получим представление для $\sigma(\mathscr{L})$ в виде $(15)$. Теорема доказана.

СЛЕдСТвиЕ 1 (теорема Массера [5]). Дифференииальньй оператор $\mathscr{L}=$ $-d / d t+a(t): D(\mathscr{L}) \subset C(\mathbb{R}, \mathbb{C}) \rightarrow C(\mathbb{R}, \mathbb{C})$, әде $a: \mathbb{R} \rightarrow \mathbb{C}$ - непрерьвная почти периодическая функция, обратим тогда и только тогда, когда $\operatorname{Re} a_{0} \neq 0$, где $a_{0} \in \mathbb{C}$ - среднее значение функиии $а$.

СЛЕДСТВИЕ 2. Если $\mathscr{U}\left(s, s-t_{0}\right), s \in \mathbb{R},-$ обратимые операторьи из End $X$ nри некотором $t_{0}>0 u \sup _{s \in \mathbb{R}}\left\|\mathscr{U}\left(s, s-t_{0}\right)^{-1}\right\|<\infty$, mо полугруппа $\left\{T_{\mathscr{U}}(t), t \geqslant 0\right\}$ вкладьввается в некоторую сильно непрерьвнуюю $\left(\right.$ при $\mathscr{F}=L_{p}$, $p \in[1, \infty)$, или $\left.\mathscr{F}=C_{0}\right)$ группу операторов и имеет место включение

$$
\sigma\left(\mathscr{L}_{\mathscr{U}}\right) \subset\left\{\lambda \in \mathbb{C}: \chi_{-}(\mathscr{U}) \leqslant \operatorname{Re} \lambda \leqslant \chi_{+}(\mathscr{U})\right\}
$$

zде $\chi_{-}(\mathscr{U})=-\lim _{\tau \rightarrow \infty} \tau^{-1} \ln \sup _{s \in \mathbb{R}}\|\mathscr{U}(s, s+\tau)\| u \mathscr{U}(s, s+\tau)=\mathscr{U}(s+\tau, s)^{-1}$ npu $\tau>0$.

Линейный оператор $\mathscr{L}_{\mathscr{U}}: D\left(\mathscr{L}_{\mathscr{U}}\right) \subset \mathscr{F} \rightarrow \mathscr{F}=\mathscr{F}(\mathbb{R}, X)$ назовем периодическим (периода $\omega>0)$, если он перестановочен с оператором $S(\omega) \in$ End $\mathscr{F}$ или, что эквивалентно, имеют место равенства $\mathscr{U}(t+\omega, s+\omega)=\mathscr{U}(t, s)$ для любых $s \leqslant t$.

ТЕОрема 6. Пусть $\mathscr{L}_{\mathscr{U}}$ является периодическим (периода 1) оператором. Тогда имеет место равенство

$$
\exp \sigma\left(\mathscr{L}_{\mathscr{U}}\right) \backslash\{0\}=\{\lambda \in \mathbb{C}: \exists \mu \in \sigma(\mathscr{U}(1,0)) \quad c|\mu|=|\lambda|\} .
$$

В частности, оператор $\mathscr{L}_{\mathscr{U}}$ обратим тогда и только тогда, когда выполнено условие $\sigma(\mathscr{U}(1,0)) \cap \mathbb{T}=\varnothing$.

ДокАЗАТЕЛЬСТво. Поскольку $\mathscr{U}(n, n-1)=\mathscr{U}(1,0)$ для любого $n \in \mathbb{Z}$, то утверждение теоремы непосредственно следует из теорем 2 и 3 (если учесть, что спектр разностного оператора $(U x)(n)=\mathscr{U}(1,0) x(n-1)$ совпадает с множеством из правой части равенства (16)). Теорема доказана.

СлЕдСТВИЕ. Если $A$ - производящий оператор сильно непрерьвной полугруппь операторов $\{T(t), t \geqslant 0\} \subset$ End $X$, mо дифференииальньй операmор $\mathscr{L}=-d / d t+A: D(\mathscr{L}) \subset \mathscr{F}(\mathbb{R}, X) \rightarrow \mathscr{F}(\mathbb{R}, X)$ обратим тогда и только тогда, когда выполнено условие $\sigma(T(1)) \cap \mathbb{T}=\varnothing$. В частности, если $X-$ гильбертово пространство, то $\mathscr{L}$ обратим только при выполнении условий (1) $\sigma(A) \cap i \mathbb{R}=\varnothing,(2) \sup _{\lambda \in \mathbb{R}}\left\|(A-i \lambda I)^{-1}\right\|<\infty$.

ДокАЗАТЕльство. Если $X$ - гильбертово пространство, то из равенства Парсеваля в гильбертовом пространстве $L_{2}(\mathbb{R}, X)$ следует, что выполнение двух приведенных условий эквивалентно обратимости оператора $\mathscr{L}=-d / d t+A$ : $D(\mathscr{L}) \subset L_{2}(\mathbb{R}, X) \rightarrow L_{2}(\mathbb{R}, X)$. Осталось использовать теорему 4. Следствие доказано.

Отметим, что непосредственно из следствия вытекает известная теорема Герхарда $[12$, с. 95$]$.

Теорема 7. Пусть семейство $\mathscr{U} \subset$ End $X$ допускает экспоненииальную дихотомию с показателем $\beta>0$ икоэффиииентом $M>0$. Если семей- 
ство эволючионных операторов $\mathscr{V}=\{\mathscr{V}(t, s), s \leqslant t\}$ из End $X$ удовлетворяет условию

$$
\sup _{n \in \mathbb{Z}}\|\mathscr{U}(n, n-1)-\mathscr{V}(n, n-1)\|<\frac{1}{M} \frac{1-\gamma}{1+\gamma},
$$

әде $\gamma=\exp (-\beta)$, mo оператор $\mathscr{L}_{\mathscr{V}}: D\left(\mathscr{L}_{\mathscr{V}}\right) \subset \mathscr{F} \rightarrow \mathscr{F}$ обратим.

ДокАЗАтЕльство. Из теорем 3 и 4 следует обратимость оператора $\mathscr{D}_{0} \in$ End $\mathscr{F}(\mathbb{Z}, X)$, а из формулы (13) получаем оценку $\left\|\mathscr{D}_{0}^{-1}\right\| \leqslant \sum_{k \in \mathbb{Z}} M \gamma^{|k|}=$ $M(1+\gamma)(1-\gamma)^{-1}$. Следовательно, из условия (17) вытекает обратимость разностного оператора $\left(\mathscr{D}_{0}^{\prime} x\right)(n)=x(n)-\mathscr{V}(n, n-1) x(n-1)$. Обратимость оператора $\mathscr{L}_{\mathscr{V}}$ следует из теоремы 3 . Теорема доказана.

\section{$\S 2$. Обсуждение полученных результатов. Примеры и замечания ${ }^{2}$}

Полученные результаты (особенно теоремы 1-4) позволяют широко использовать теорию полугрупп линейных операторов при исследовании линейных параболических дифференциальных операторов с переменными коэффициентами и, следовательно, для дифференциальных операторов с частными производными. Например, к рассматриваемым операторам относится дифференциальный опеpaтор

$$
\mathscr{L}=-d / d t+A(t): D(\mathscr{L}) \subset L_{p}(\mathbb{R}, X) \rightarrow L_{p}(\mathbb{R}, X),
$$

где $X=L_{2}(\Omega)=L_{2}(\Omega, \mathbb{C}), \Omega$ - ограниченная гладкая область из $\mathbb{R}^{n}$. Семейство линейных дифференциальных операторов $A(t): H_{0}^{m}(\Omega) \cap H^{2 m}(\Omega) \subset$ $L_{2}(\Omega) \rightarrow L_{2}(\Omega), t \in \mathbb{R}\left(H_{0}^{m}(\Omega), H^{2 m}(\Omega)\right.$ - пространства Соболева [6]), определяется семейством дифференциальных выражений

$$
\left(l_{t} y\right)(u)=\sum_{|\alpha| \leqslant 2 m} a_{\alpha}(t, u)\left(D^{\alpha} y\right)(u), \quad u \in \Omega, t \in \mathbb{R},
$$

и краевыми условиями Дирихле на границе $\partial \Omega$ области $\Omega$. Функции $a_{\alpha}: \mathbb{R} \times \Omega \rightarrow$ $\mathbb{C},|\alpha| \leqslant 2 m$, считаются принадлежащими пространству $C\left(\mathbb{R}, C^{k}(\Omega)\right)$ для достаточно большого $k \in \mathbb{N}$ и удовлетворяют условию Липшица, если их рассматривать как функции от первого аргумента со значениями в $C^{k}(\Omega)$. Кроме перечисленных условий предполагается, что семейство дифференциальных выражений $l_{t}, t \in \mathbb{R}$, равномерно эллиптично.

Из приведенных условий следует, что эллиптические операторы $A(t), t \in \mathbb{R}$, являются производящими операторами аналитических полугрупп линейных ограниченных операторов, причем выполнены условия теоремы Соболевского-Танабе [13, с. 589], которая гарантирует корректность задачи Коши на $\mathbb{R}$ (существование семейства эволюционных операторов).

В заключение отметим, что в работах $[5,9,10]$ рассматривались дифференциальные операторы вида $-d / d t+A(t)$ в банаховом пространстве $C(\mathbb{R}, X)$ с функцией $A: \mathbb{R} \rightarrow$ End $X$. Утверждение (1) теоремы 4 было получено в монографии [10, теорема $\left.3.3^{\prime}\right]$ при некоторых дополнительных условиях, которые для пространства $\mathscr{F}=C$ были сняты в работе $[1$, гл. $\mathrm{X}]$, причем там не требовалось выполнение условия $A(t) \in \operatorname{End} X, t \in \mathbb{R}$. В статье [9] было доказано, что условие экспоненциальной дихотомии семейства эволюционных операторов

\footnotetext{
${ }^{2}$ Материал этого параграфа представлен в редакцию журнала 6 декабря 1995 г.
} 
эквивалентно выполнению условия $\sigma\left(T_{\mathscr{U}}(1)\right) \cap \mathbb{T}=\varnothing$. Аналогичное утверждение приведено в монографии [15, теорема 9.3] для элементов некоторых $C^{*}$-алгебр, порожденных динамическими системами.

Дифференциальные операторы с неограниченными операторными коэффициентами рассматривались в работах $[1,6,8]$. В статье [8] была доказана равносильность условий (равномерной) инъективности операторов $\mathscr{L}_{\mathscr{U}}$ и $\mathscr{D}_{0}$. Среди имеюшихся в монографии [6] результатов, которые наиболее связаны с теоремами 1-4, отметим теорему 7.6.3, в которой установлена обратимость оператора $\mathscr{L}_{\mathscr{U}}$ при некоторых условиях, включающих наличие экспоненциальной дихотомии семейства $\mathscr{U}$, и утверждение (см. с. 251) об экспоненциальной дихотомии этого семейства при условии дискретной дихотомии семейства операторов $\left\{\mathscr{U}\left(t_{0}+n, t_{0}+n-1\right), n \in \mathbb{Z}\right\}$ для всех $t_{0} \in \mathbb{R}$. Следует отметить, что из $[6$, теорема 7.6.5] и теорем 3 и 4 следует, что семейство $\mathscr{U}$ допускает экспоненциальную дихотомию, если допускает дискретную дихотомию лишь семейство $\{\mathscr{U}(n, n-1), n \in \mathbb{Z}\}$. Дискретный аналог утверждения 1 теоремы 4 имеется в [6, теорема 7.6.5], и там же (на с. 363 в комментариях к гл. IX) ставился вопрос о доказательстве утверждения 1 теоремы 4.

\section{ЛитеРАТУРА}

1. Левитан Б. М., Жиков В. В. Почти периодические функции и дифференциальные уравнения. Изд-во МГУ, М., 1978.

2. Крейн C. Г. Линейные дифференциальные уравнения в банаховом пространстве. Наука, М., 1967.

3. Хилле Э., Филлипс Р. Функциональный анализ и полугруппы. ИЛ, М., 1962.

4. Baskakov A. G. Investigation of spectral properties of differential operator $\mathscr{L}=$ $-d / d t+A(t)$ by means of semigroup $\exp \mathscr{L} t, t \geqslant 0$. Intern. Conf. on Functional Equations and Application, Moscow, Russia, August 14-21 (1994), p. 8-9.

5. Массера $X$. Л., Шеффер $X . X$. Линейные дифференциальные уравнения и функциональные пространства. Мир, М., 1970.

6. Хенри Д. Геометрическая теория полулинейных параболических уравнений. Мир, М., 1985.

7. Баскаков А. Г. Спектральные критерии почти периодичности решений функциональных уравнений. Матем. заметки, 24, №2, 195-205 (1978).

8. Тюрин B. M. Об обратимости линейных дифференциальных операторов в некоторых функциональных пространствах. Сиб. матем. ж., 32, №3, 160-165 (1991).

9. Забрейко П. П., Нууен Ван Минь. Группа характеристических операторов и ее применения в теории линейных обыкновенных дифференциальных операторов. Доклады РАН, 324, №1, 24-28 (1992).

10. Далеикий Ю. Л., Крейн М. Г. Устойчивость решений дифференциальных уравнений в банаховом пространстве. Наука, М., 1970.

11. Баскаков А. Г. Абстрактный гармонический анализ и асимптотические оценки элементов обратных матриц. Матем. заметки, 52, №2, 17-25 (1992).

12. Nagel $R$. One-parameters semigroups of positive operators. Lecture Notes in Math., Vol. 1184, Springer-Verlag, 1984.

13. Иосида К. Функциональный анализ. Мир, М., 1967.

14. Данфорд Н., Швари Дж. Т. Линейные операторы. Т. 1, ИЛ, М., 1962.

15. Антоневич А. Б. Линейные функциональные уравнения: Операторный подход. Университетское, Минск, 1988. 